\title{
Optical Excitation and Probing of Bottle Microresonators
}

\author{
G. Senthil Murugan, J. S. Wilkinson \& M. N. Zervas \\ Optoelectronics Research Centre, University of Southampton, UK \\ *mnz@orc.soton.ac.uk
}

Keywords: Microresonators, Fibres, Filters, Add-Drop Multiplexers

Novel bottle microresonators [1] fabricated from standard telecommunications optical fibers were recently shown to support helical whispering gallery modes (WGMs) extending along the bottle length, between the bottle necks. Intensity maxima were observed around the turning points on both sides close to the bottle-necks where the WGMs are effectively reflected [2].

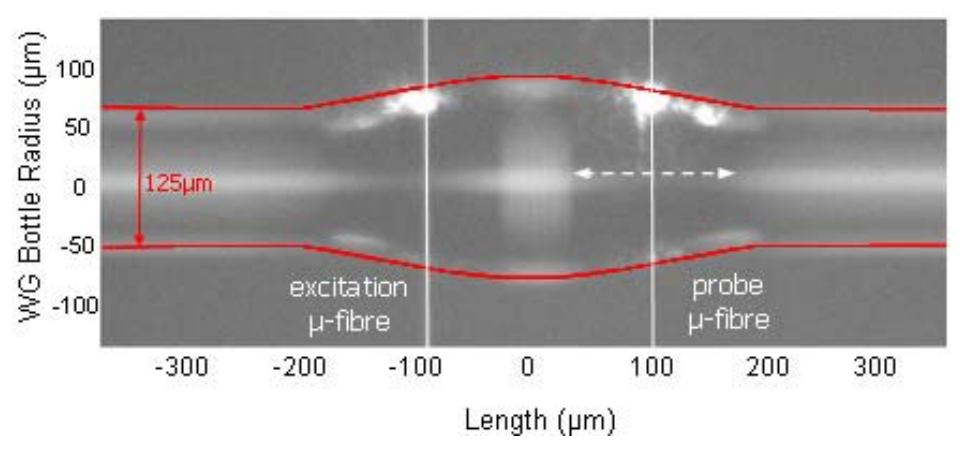

Figure 1: Image of bottle microresonator with excitation and probe tapered optical fibres placed at $100 \mu \mathrm{m}$ to the left and right of the microbottle centre, respectively.
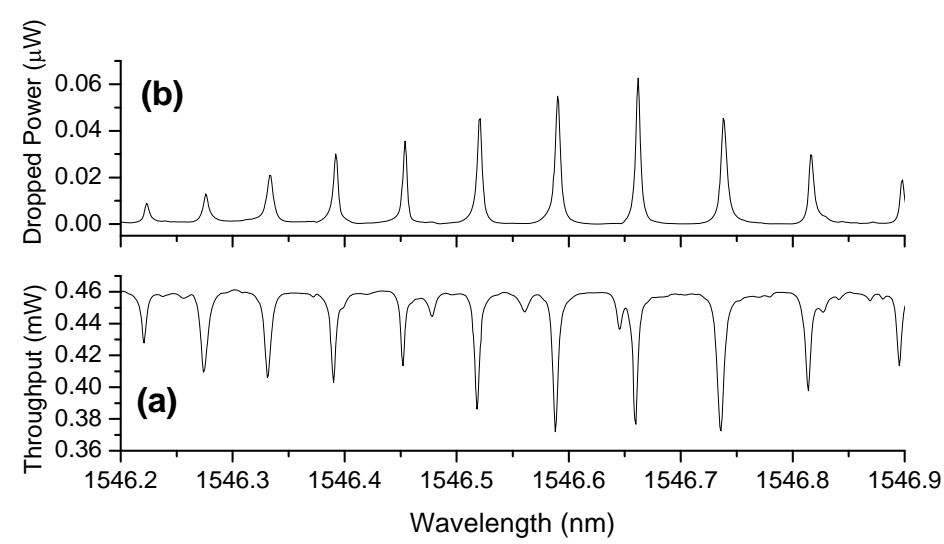

Figure 2: (a) throughput and (b) dropped power when the bottle microresonator was excited and probed at $50 \mu \mathrm{m}$ to the left and right of the microbottle centre, respectively.
Selective excitation on one side of the bottle microresonator leads to strong power localization at two symmetrically-placed turning points for the WGMs and can be potentially exploited to form effective add-drop filters. A tapered optical fiber (2-3 microns in diameter with effective index of about 1.2) was placed on one side of the bottle to excite the bottle WGMs. A similar tapered fiber placed symmetrically on the other side of the bottle acted as a probe to extract the excited modes. The probe micro-fibre was shifted along the microbottle length and its power transmission was monitored, in order to investigate the WGM modal excitation efficiency.

Figure 1 shows scattered light at the points of maximum intensity of a characteristic helical WGM, supported by the bottle microresonator, when the excitation micro-fibre is placed at $100 \mu \mathrm{m}$ to the left of the microbottle centre.

Channel dropping characteristics have been studied for the first time in this novel type of microresonator. We have successfully extracted power from all the resonance wavelengths using the probe placed at an appropriate position on the bottle. Figure 2 shows the throughput of the excitation fibre (a) and the power collected by the probe (b), when excitation and probe micro-fibres are placed symmetrically at $50 \mu \mathrm{m}$ from the microbottle centre. A series of spectral data were taken with the excitation and probe micro-fibres at different locations along the microbottle length. The results will be presented and discussed in detail at the conference.

The results given in Figure 2 show that microbottle resonators can be potentially used for the implementation of efficient add-drop multiplexers. By properly matching the effective indices of the excitation and probe tapered fibers with the bottle microresonator and satisfying the critical coupling conditions, a highly efficient add-drop filter can potentially be realised.

Acknowledgments: The authors thank Dr. Yongmin Jung for providing the tapered fiber coupler and Dr. Yuh Tat Cho for providing the fusion splicer. This work was funded by the UK EPSRC under grant GR/S96500/01.

\section{References}

[1] M. Sumetsky, "Whispering-gallery-bottle microcavities: the three-dimensional etalon," Opt. Lett. 29, 8-10 (2004).

[2] G. Senthil Murugan, J. S. Wilkinson \& M. N. Zervas, "Selective excitation of whispering gallery modes in a novel bottle microresonator", Optics Express Vol.17 pp.11916-11925 (2009). 